\title{
Cuestiones teóricas para la enseñanza de la vaguedad lingüística ${ }^{1}$
}

\author{
ADRIÀ PARDO LliBRER \\ Universitat de València \\ adria.pardo@uv.es
}

\begin{abstract}
Resumen: Este artículo estudia, desde un punto vista teórico, el problema de la vaguedad lingüística con vistas a posibles aplicaciones en el aula de E/LE. De entrada, se define la noción de vaguedad lingüística; tanto desde una óptica filosófica (predicados difusos), como lingüística (la existencia de ciertos términos que modifican los valores de verdad de una proposición). Así, se sintetizan las principales teorías de la vaguedad, estableciendo una distinción operativa entre usos semánticos y pragmáticos de los denominados vaguificadores. Dichos usos pragmáticos pertenecen al nivel discursivo y funcionan dentro de estrategias propias de la atenuación lingüística. Pensamos que estas indicaciones modulares no son incompatibles con la enseñanza de E/LE, sino que pueden constituir una técnica que contribuya a la capacidad metalingüística del alumnado.
\end{abstract}

Palabras clave: vaguedad lingüística, vaguificadores, adverbios, léxico bicategorial

\section{Theoretical remarks on the teaching of linguistic vagueness}

Abstract: This paper explores several theoretical frameworks of linguistic vagueness, in order to outline further learning applications. In the first place, I deal with the definition of linguistic vagueness, understood either as a philosophical problem (fuzzy predicates) or a as linguistic problem (regarding the truth-conditions of a given proposition). In this sense, I address a comprehensive review of some theories on vagueness, establishing a distinction between semantic and pragmatic uses of the socalled vagueifiers. Ultimately, pragmatic uses of vagueifiers belong to the discourse level, involving hedging strategies. This distinction can be applied for teaching the Spanish vague language, implementing the students' metalinguistic tools as well.

Key words: linguistic vagueness, vagueifiers, adverbs, lexical bicotegorial items

\section{Introducción}

La vaguedad lingüística (en adelante, $\mathrm{VL}$ ) es un concepto amplio: engloba desde trabajos en filosofía del lenguaje (Williamson 1994) hasta análisis de corte semántico (Lakoff 1973) o pragmático (Kaltenböck et ál. 2010), así como ciertos estudios aplicados (Prince et ál. 1980; Crissmore et ál. 1993 entre otros). Este artículo revisa, en primer lugar $(\S 2)$, las diferentes perspectivas sobre la VL, centrándose en una noción propiamente lingüística. Seguidamente $(\S 3)$, se introducen los vaguificadores: aquellos elementos que predican, de proposiciones definidas, denotaciones indeterminadas. Los

\footnotetext{
${ }^{1}$ Este artículo ha sido posible gracias al proyecto de investigación FFI2016-77842-P, Unidades discursivas para una descripción sistemática de los marcadores del discurso en español (UDEMADIS), financiado por el MINECO, la AEI y los fondos FEDER.
} 
vaguificadores oscilan entre los niveles semántico y pragmático, por lo que son clave para la comprensión de la VL. Así, es posible trasladar la VL al aula de E/LE, si el docente dispone de criterios adecuados mediante los cuales puedan identificarse aquellas funciones específicamente discursivas (§ 4). Las conclusiones cierran el artículo $(\S 5)$.

\section{Vaguedad lingüística: conceptos y aplicaciones}

La VL hace referencia al lenguaje vago. Por lenguaje vago suele entenderse un uso impreciso del lenguaje. Esta concepción tan evidente resulta, sin embargo, limitada. La VL se concibe de manera diferente según la disciplina implicada (filosofía, lingüística), la rama de dicha disciplina (semántica, pragmática) o su marco teórico (formal, funcional). Una situación que se complica al considerar posibles aplicaciones docentes. Por ello, este apartado sintetiza los tres principales enfoques de la VL. En primer lugar (§ 2.1), se introduce la concepción filosófico-formal de la VL. Frente a esta visión analítica de la VL, se tratan los estudios sobre hedging (§ 2.2), una investigación propiamente lingüística centrada en formas y estructuras que modifican el significado y/o la función de una frase. Por último ( $§ 2.3)$, se presentan las dos sistematizaciones en torno a la VL que, pensamos, mejor pueden esbozar una primera propuesta didáctica.

\subsection{La concepción filosófico-formal de la vaguedad lingüística}

La filosofía de la VL se define como el conjunto de estudios deductivos (lógica y filosofía) centrados la descripción de proposiciones cuya formulación no permite discernir, en principio, un límite claro entre verdad o falsedad. La bibliografía sobre esta temántica es extensa y escapa a los objetivos de este trabajo (Williamson 1994; Keefe and Smith 1997, Keefe 2003 entre otros); sin embargo, es importante introducir el fenómeno filosófico de vaguedad y su relación con el hecho lingüístico.

Puede decirse que «an expression is vague if it admits borderline cases» (Bach 1998: 12). Algunos ejemplos clásicos son predicados como 'calvo' o 'alto', que dan lugar a proposiciones vagas. Tres son sus características: «they admit borderline cases, they lack (or at least aparently lack) sharp boundaries and they are susceptible to sorites paradoxes» (Keefe 2003: 9). Esta definición filosófica de VL está bastante simplificada, pero se adecúa a los objetivos de este apartado introductorio:

a. Casos fronterizos-. En una proposición como Este caqui es rojo, el término 'rojo' es difuso, pues puede aplicarse a un continuo cromático que abarca desde 'rojo-anaranjado' hasta 'rojo-bermellón'. Según Keefe (2003: 6), el decir Este caqui es rojo y no es rojo revela la ausencia de un frontera con que juzgar algo como 'definitivamente rojo', cancelando el principio lógico de bivalencia.

b. Ausencia de límites precisos-. Las proposiciones vagas presentan límites difusos. Considérese, para el predicado vago 'alto' en una proposición dada (Pau Gasol es alto), una supuesta escala de altura (p. ej. estatura media de la NBA), y predíquese de otro argumento: Gasol es alto y así lo es mi amigo 
pigmeo. Si tal combinación no puede desambiguarse (vid. Sadock y Zwicky 1975), no existe un grado concreto para discernir verdad o falsedad en 'alto' 2 .

c. Susceptibles a la paradoja sorites-. La paradoja sorites plantea el siguiente problema: «a heap of sand consists of only ifnitely many grains, but when they are carefully removed one by one, we have no idea how to answer the question When did there cease to be a heap?» (Williamson 2007: 33). En otras palabras, solo en función de cuántos pelos de más o de cuántos pelos menos tenga un individuo, la predicación definitiva de 'calvo' es indetectable.

En suma, el problema filosófico de la VL es el problema de «being unable [...] to say of any single point along an ordering generated by the meaning of a particular point whether that point separates the thing that the term is true [or false] of» (Fara y Russel 2012: 337). De proposiciones como Pepe está calvo o Luis es joven se desprenden cuestiones del tipo: ¿a partir de cuántos pelos alguien es calvo? o ¿hasta qué punto una persona es joven y a partir de qué punto pasa a ser vieja? Esta es, en resumen, la cuestión de fondo de la filosofía de la VL (desarrollada mediante metalenguaje lógico).

Si bien la VL y la vaguedad filosófica remiten a disciplinas diferentes, la primera participa en cierto modo de la segunda (Cintula et ál. 2011). Ello reduce su metalenguaje al estudio de proposiciones ad hoc, persistiendo como propiedad general del lenguaje, pero no proporciona una descripción lingüística completa.

\subsection{El estudio del fenómeno del hedging}

Si la filosofía del lenguaje mantiene una perspectiva formal, los estudios sobre el hedging conciben la VL una categoría semántico-pragmática. Estos estudios atienden a los diferentes modos (elecciones léxicas, derivación, estructuras sintácticas, etc.) en que el fenómeno vago se manifiesta en una lengua en concreto. El término hedging deriva de su objeto de estudio: los denominados hedges. La primera definición de hedge provenie de Lakoff (1973: 95): «those words whose labor is to make things fuzzier or less fuzzy». Así, si incorporamos a una frase como (1) un hedge como el de (2):

(1) Mateo cobra $1000 €$ al mes

(2) Mateo cobra más o menos $1000 €$ al mes

Se produce una modificación del contenido proposicional, no porque la proposición (1) contenga un predicado intrínsecamente vago (como 'calvo' o 'alto'), sino porque la presencia del adverbio más o menos (2) suspende la asignación de valores de verdad:

(1') Mateo cobra $1000 €$ al mes: verdadero si y solo si cobra $1000 €$, si no, falso

(2’) Mateo cobra más o menos $1000 €$ al mes: puede cobrar y puede no cobrar $1000 €$

De acuerdo con Lakoff (1973), proposiciones como (2’) no se ajustan a una lógica de proposiciones bivalente, sino que, en todo caso, su análisis requiere una lógica modal:

(2') Mateo cobra más o menos $1000 €$ al mes: 'más-o-menos-P' : $\diamond \mathrm{P} \rightarrow\left(\mathrm{P}^{\mathrm{V}} \neg \mathrm{P}\right)$

\footnotetext{
${ }^{2}$ Esto podría cuestionar que Pau Gasol es alto sea una proposición bien formada, puesto que «according to classical logic and semantics all predicates have well-defined extensions» (Keefe y Smith 1997: 3).
} 
Esta notación se ajustaría, asimismo, a otros hedges propios del lenguaje natural:

(3) Mateo tiene el pelo tipo rubio

(4) Mateo se puso en plan filosófico

Al reformular estos ejemplos, resulta imposible afirmar que sea del todo falso o del todo verdadero el hecho de que Mateo tenga el pelo rubio o de que sea un filósofo como tal:

(3') Mateo tiene el pelo tipo rubio, o sea, entre rubio y castaño

(4') Mateo se puso en plan filosófico, vamos, que se las daba de filósofo

Estas alteraciones del significado no dependen de un predicado cuyos límites epistemológicos son difíciles de delimitar: proposiciones como (1) son claramente susceptibles de recibir valores de verdad, pero la modificación por un adverbio (2) imposibilita el análisis semántico de una proposición en principio bien formada (2'). Desde un punto de vista lingüístico, la VL reproduce en una proposición las propiedades de los predicados difusos mediante los mecanismos léxicos de que dispone la lengua. De esta manera, junto a los hedges (entendidos como formas) existen también diferentes estructuras sintácticas que participan de la VL. Es el caso de los hedged performatives:

(5) Creo que a Mateo le hace tilín su compañera de clase

Los hedged performatives son compuestos sintácticos funcionalmente equivalentes a los hedges (Fraser 1980, 1975; Mihatsch 2013), como estructuras marco o parentéticos (Dehé y Kavalova 2007; Kaltenböck 2007). La función discursiva añadida, por la cual el hablante reduce la fuerza ilocutiva del enunciado, se da en la cláusula principal (Creo que), si bien esta es informativamente secundaria (Simon-Vandenbergen 2000).

Bajo la categoría hedging se incluyen hedges y hedged performatives, en la medida en que son funcionalmente similares y (aunque morfosintácticamente diferentes) alteran el significado de una proposición con vistas a una función pragmático-discursiva. Por ello, el hedging suele definirse como «a pragmatic function that involves all levels of linguistic analysis» (Kaltenböck et ál. 2010: 3). Para organizar esta variedad de conceptos, dentro de la teoría de la mitigación existe una tipología de hedges, basada en la distinción establecida por Prince et ál. (1980). Según Caffi (1999, 2007), pueden distinguirse tres tipos de hedges en función del nivel de análisis sobre el que actúan:

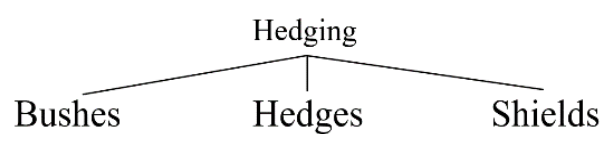

Figura 1. Modelo ternnario

Los bushes son hedges en el sentido de Lakoff (1973), alterando las condiciones de verdad. Los hedges son formas unilexemáticas que reducen la fuerza ilocutiva. Los shields, por último, son expresiones complejas cuya función es pragmática (esto es, como los hedged performatives). Este modelo ternario (Caffi 2007) evidencia la 
confluencia de niveles lingüísticos en elementos característicos de la VL, como son los hedges. Si los bushes son elementos semánticos y los shields pragmáticos, los hegdes están a caballo entre significado y uso, cuya frontera debe ser delimitada, pues son los que se prestan a mayor confusión a la hora de ser explicados a estudiantes de español.

\subsection{Teorías lingüísticas sobre la vaguedad}

Frente a los estudios sobre hedging, existen dos teorías que proponen una sistematización para la VL. Por un lado, en la tradición anglosajona (Wachtel 1980; Channell 1981), Channell (1984) establece una tipología de formas de VL, con vistas no solo al análisis lingüístico en general, sino también a la enseñanza de lenguas extranjeras (Channell 1985). Por otro lado, la lingüística italiana (Voghera 2017) plantea una distinción de base pragmática entre vaguedad sistémica e intencional, así como de los tres subtipos de esta última. A continuación, se exponen ambas teorías.

En primer lugar, Channell (1994: 17) define VL como los «different ways in which speakers can avoid being precise». La orientación de su trabajo es formal, pues propone una relación de categorías discretas mediante las cuales describir las diferentes estrategias lingüísticas para la VL. Estas estrategias son dependientes del discurso y la situación comunicativa, sin embargo, es posible enumerarlos como sigue:

\begin{tabular}{|l|l|l|}
\hline \multirow{2}{*}{ Aproximadores de cantidad numérica } & adverbios & aproximadamente... \\
\cline { 2 - 3 } & estructuras recurrentes & seis o así... \\
\cline { 2 - 3 } & especificadores & al menos... \\
\hline Aproximación numérica con número redondo & a 10 metros, unos 30 años... \\
\hline Aproximadores no-numéricos de cantidad & montón de, un par... \\
\hline Referencia vaga a categorías & o algo... \\
\hline Proformas & cosa trasto... \\
\hline
\end{tabular}

Figura 2. Recursos formales de la vaguedad lingüística

En segundo lugar, la teoría de la vaghezza se apoya en las categorías de Channell (1994) para la descripción de la VL, pero plantea una distinción de base pragmática. Así, en primer lugar, Voghera (2017) distingue vaguedad sistémica de vaguedad intencional. En la vaguedad sistémica se incluyen predicados con límites epistemológicamente difusos:

(6) Pau Gasol es alto $\approx$ Pau Gasol es más o menos alto

(7) Mateo cobra más o menos $1000 €$ al mes $\neq$ Mateo cobra $1000 €$ al mes

Frente a enunciados cuya vaguedad resulta de la naturaleza difusa de uno de sus predicados (6), los enunciados que incorporan un elemento léxico, pero cuya ausencia produciría un enunciado neutro (7), reflejan una intención vaga por parte del hablante. Dentro de esta vaguedad intencional existen tres subtipos:

1. Vaguedad informativa-. La vaguedad informativa se deriva de la falta de conocimiento sobre un significado, generando una proposición vaga. Son los elementos cuya principal repercusión tiene lugar en el nivel semántico. 
2. Vaguedad relacional-. Remite a la relación entre el hablante e interlocutor. Distingue aquellas formas cuyo nivel de actuación no diferencia claramente entre modificadores semánticos y aquellas funciones semántico-ilocutivas ${ }^{3}$.

3. Vaguedad enunciativa-. Se relaciona con la elaboración espontánea del habla. Se trata de aquellos elementos formulativos que no comprometen la imagen del hablante pese a la ausencia de planificación del discurso hablado.

\section{Los vaguificadores}

Llegados a este punto, pueden concluirse tres aspectos clave de la VL: a) cada lengua dispone de mecanismos determinados para dotar de vaguedad los enunciados, $b$ ) estos enunciados responden a una intención por parte del hablante a la hora de denotar vaguedad, $c$ ) es posible acotar el estudio de la VL si disponemos de parámetros formales para su descripción. Los elementos formales en que se centra este trabajo son aquellas elecciones léxicas que modifican las condiciones de verdad de una proposición, al tiempo que presentan desarrollos pragmáticos en el nivel del enunciado. Por ejemplo:

(8) A lo mejor voy a tu fiesta: 'a lo mejor-P' $: \diamond \mathrm{P} \rightarrow\left(\mathrm{P}^{\mathrm{V}} \neg \mathrm{P}\right)$

$$
\left(\mathrm{Q}^{+>}\right) \text {no-seguro-P : (++>) no-posible compromiso }
$$

El uso del adverbio a lo mejor en (8) presenta dos niveles de análisis. Por un lado, el semántico $\left(\diamond \mathrm{P} \rightarrow\left(\mathrm{P}^{\mathrm{V}} \neg \mathrm{P}\right)\right)$ : impidiendo una asignación de valores de verdad ('puede que vaya y puede que no vaya'). Por otro lado, el pragmático: con base en un significado de modalidad dubitativa, el hablante se vale de una implicatura de base $Q$ (Levinson 2000) a fin de proteger su imagen ante una respuesta despreferida (Albelda 2010).

Estos elementos son los que Eklund (2001, 2005) denomina vaguificadores (vagueifiers); es decir, términos que introducen vaguedad en una proposición. Existen otros mecanismos que, desde un punto de vista lingüístico, contribuyen a la expresión de la vaguedad, como ciertas formas de derivación con función atenuadora (Briz 1995) o determinadas entonaciones ascendentes (Hidalgo 2013). Sin embargo, estos fenómenos (claramente morfosintácticos o prosódicos), a diferencia de lo que ocurre con los vaguificadores, no se prestan a una confusión de niveles $(\S 3.1)$ ni se confunden sus funciones dada una coincidencia morfológica con otros elementos $(\S 3.2)$.

\subsection{Problemas fronterizos}

Los vaguificadores oscilan entre los niveles semántico y pragmático: un vaguificador (8) puede tener funciones pragmáticas secundarias, pero este uso discursivo depende de un significado cuyo contenido proposicional está previamente vaguificado. En otras palabras, los vaguificadores responden (en términos de Voghera, 2017) a una VL de tipo informativa: constituyen un hedge que altera el valor de verdad de la proposición y que, en segundo término (8a), repercute en la fuerza ilocutiva de un enunciado ( $8 b$ ). Esta situación, pues, plantea un problema a la hora de enseñar el funcionamiento de este

\footnotetext{
${ }^{3}$ La vaguedad relacional primaría la consideración de hedges por tratarse de elementos interpersonales.
} 
tipo de formas a estudiantes de español (normalmente englobadas en el cajón de sastre de la categoría adverbio). Se plantean así tres posibles soluciones para su tratamiento:

a. Los vaguificadores como adverbios: esta solución es formalmente la más cómoda, ya que permite elaborar listados de términos en L2 con una cierta equivalencia en la L1 del estudiante, si bien obvia los usos pragmáticos.

b. Los vaguificadores como marcadores del discurso: esta postura contempla los usos pragmáticos (mitigación, atenuación, etc.); sin embargo, en la mayoría de casos son usos subsidiarios del significado proposicional, lo que puede inducir al estudiante a un empleo erróneo en el nivel semántico.

c. Las vaguificadores como elementos léxicos bicategoriales: por elementos léxicos bicategoriales (en adelante, ELB) pueden entenderse las formas que, en función del nivel lingüístico sobre el que actúen, deben analizarse como adverbios (con repercusión semántica) o como marcadores del discurso (extraproposicionales y ajenos a la estructura de la oración).

\subsection{Los vaguificadores como elementos léxicos bicategoriales}

Ciertos vaguificadores, como a lo mejor o más o menos no pueden recibir una función biunívoca: pueden responder a dos categorías diferentes y, por lo tanto, su clasificación no puede ser en ningún caso automática. Esta situación se aprecia claramente en muestras del habla coloquial ${ }^{4}$, adscribiéndose en unos casos a la categoría adverbial (910) y en otros casos a la categoría de marcador del discurso (11-12):

(9) $260 \mathrm{~B}:[\mathrm{no} /$ pero] es que normalmente antes $\uparrow$ no sé/ a lo mejor estoy equivocao/ $[\mathrm{peroo} / / \mathrm{pues} \rightarrow=]$

(10) $133 \mathrm{~B}:=\underline{\text { o sea sí sabes valenciano más o menos }} \rightarrow \S$

(11) $17 \mathrm{~A}:=$ es eso/ yo a lo mejor puedo hacer un proyecto $\uparrow /$ que para mí sea $\uparrow /$ superbién $\uparrow$ que yo diga $\uparrow$ / es que veo que así/ utilizo más el espacio $\uparrow$ o utilizo más el tiempo $\uparrow$ o está mejor aprovechado/ o veo que hay una materia que es mucho más fácil de- dee- de sacar $\uparrow$ o que se le [puede $\uparrow=$ ]

(12) 232E: pero ((com))- para una vez que tengo dinero / para una vez que tengo dinero $\uparrow /$ más o menos pa gastar $\uparrow \S$

Los usos (9-10) son adverbiales: si se eliminan, se generan proposiciones susceptibles de condiciones de verdad (9'-10'). Por su parte, (11-12) funcionan como marcadores discursivos, un uso suprasintáctico cuya elisión no modifica su significado (11 '-12'):

(9') 'a lo mejor estoy equivocado’ $: \diamond \mathrm{P} \rightarrow\left(\mathrm{P}^{\mathrm{V}} \neg \mathrm{P}\right)$

(= 'puedo estar y puedo no estar equivocado') $\neq$ ' ø estoy equivocado'

(10’) 'más o menos sabes valenciano' $: \diamond \mathrm{P} \rightarrow\left(\mathrm{P}^{\mathrm{V}} \neg \mathrm{P}\right)$

(= 'sabes y no sabes hablar valenciano' $) \neq$ ' $\varnothing$ sabes valenciano'

$(11$ ') 'yo a lo mejor puedo hacer un proyecto' : $\mathrm{P}(\approx$ 'yo ø puedo hacer un proyecto')

$(12$ ') 'tengo dinero más o menos para gastar' : $\mathrm{P}(\approx$ 'tengo dinero $\varnothing$ para gastar')

\footnotetext{
${ }^{4}$ Estas muestras de habla se obtienen del corpus Valesco 2.0 (Cabedo y Pons, 2013), de acceso en línea.
} 
La distinción categorial se da en función del nivel de análisis en que actúan. Así, los ELB son formas léxicas que, en la mayoría de casos, funcionan como adverbios (9-10), pero que también pueden presentar usos estrictamente pragmático-discursivos (11-12).

\section{Hacia una enseñanza sistematizada de la vaguedad lingüística}

En el amplio campo de la VL, la cuestión de los vaguificadores presenta unas exigencias metalingüísticas que hacen de su enseñanza en el aula de E/LE un tema especialmente complejo. Lo que sigue, pues, es un primer esbozo de posibles pautas para el docente a la hora de discernir (en tanto que ELB) los usos discursivos de los adverbiales.

\subsection{Apuntes para una distinción de planos modales}

El punto de partida para la distinción categorial de un vaguificador consiste en discernir si funciona como adverbio o como marcador del discurso. Si se trata de un adverbio, su elisión de la oración genera un notable cambio de significado (12-13):

(13) 218 B: $\S$ quee quee a lo mejor nos pasa a todas o algo [(así)]

a. NIVEL SEMÁNTICO: 'algo nos pasa (P) o no nos pasa $(\neg \mathrm{P})$ '

b. NIVEL PRAGMÁTICO: $\left(\mathrm{Q}^{+>}\right)$no es seguro que nos pase : $(++>)$atenuación

Los vaguificadores se ubican entre lo semántico y lo pragmático. Ante los evidentes desarrollos pragmáticos (13b), podría pensarse que los vaguificadores suponen un elemento discursivo. Para no incurrir en el error de obviar su uso semántico (13a), cabe describir la forma lógica mínima de la proposición en que el vaguificador se encuentra:

(13a') A lo mejor eso nos pasa a todas $(\diamond \mathrm{P}) \neq \varnothing$ eso nos pasa a todas $(\mathrm{P})$

La modificación proposicional invita a una categorización adverbial. Ahora bien, el problema de los ELB es que se prestan a una confusión de la modalidad. Esto es, presentan una modalización pragmática subsidiaria cuando funcionan en tanto que adverbio (13b), pero también se relacionan con la modalidad oracional cuando su función es la propia de un marcador del discurso (14):

(14) $28 \mathrm{P}:{ }^{\circ}(\text { no })^{\circ}$ no porque no ha ocurrido nadaa $\uparrow$ noo $\uparrow$ no se ha caido ni nadaa/ peroo a lo mejor si se hubiese caídoo// [...]

Mientras que una oración en modo indicativo (modalidad asertiva) cambia a una modalidad oracional dubitativa al incorporar a lo mejor (13), en una oración en modo subjuntivo (14) puede entenderse como un marcador del discurso, puesto que su modalización resulta en última instancia pragmática ${ }^{5}$ frente a la modalidad oracional del verbo en cuestión. Dado que a lo mejor no suspende semánticamente la aserción,

\footnotetext{
${ }^{5}$ En todo caso, se trataría de una implicatura conversacional generalizada de base $M$ (Levinson, 2000).
} 
pensamos que es precisamente en estos casos cuando su consideración como elemento discursivo es más pertinente en el marco de la enseñanza de estrategias comunicativas.

\subsection{Los vaguificadores y las estrategias de atenuación lingüística}

En conclusión, la desambiguación de los vaguificadores (en tanto que ELB) es un proceso para clasificar formas según su función. Cabe preguntarse, entonces, cuál es la utilidad de este filtrado tan exhaustivo para un profesor de E/LE.

- En primer lugar, esta distinción entre vaguificadores constituye una primera pauta mediante la cual acotar una parcela del fenómeno de la VL.

- En segundo lugar, una diferenciación sistemática de los vaguificadores permite enriquecer (en niveles superiores de aprendizaje) el dominio, no solo de la competencia lingüística (disponiendo de estrategias metalingüísticas para identificar elementos proposicionales), sino también la competencia discursiva (encontrando una utilidad práctica a fenómenos que exceden la gramática).

- Finalmente, esta competencia discursiva cobra forma especialmente en los vaguificadores que no repercuten en el significado de una oración (14').

(14') 28 P: ${ }^{\circ}(\text { no })^{\circ}$ no porque no ha ocurrido nadaa $\uparrow$ noo $\uparrow$ no se ha caido ni nadaa/ pero $\boldsymbol{\sigma}$ si se hubiese caídoo // [...]

En estos casos, una primera solución es la de su tratamiento como atenuadores lingüísticos (siguiendo a Albelda 2010).

En definitiva, la perspectiva funcional que ofrece el acercamiento de la vaguedad intencional invita replantearse las sistematizaciones de corte formal (del tipo Channell 1994; vid. § 2.2), dado que obliga a un análisis caso por caso de elementos que no se ajustan estrictamente a las habilidades gramaticales del alumnado (Pons 2005).

\section{Conclusiones}

Ante un campo de investigación tan vasto, todo acercamiento teórico a la VL obliga a una distinción entre el interés filosófico y los desarrollos lingüísticos. Dentro del área lingüística, las teorías son igualmente variadas. Tal situación sobredimensiona el tratamiento de los elementos propios de la VL. Sin embargo, es posible organizar ciertas formas léxicas relativas a la VL: los vaguificadores. Estas formas presentan tanto usos adverbiales como funciones propias de un marcador del discurso, por lo que no es posible enseñarlas dentro de listados cerrados con excepciones propias de lo coloquial. Entender los vaguificadores como ELB ayuda no solo a distinguir aquellos usos en que un hablante modifica el significado de una proposición, sino que pone al alcance del docente una herramienta añadida mediante la cual poder explicar la atenuación de enunciados ya de por sí modalizados.

\section{Bibliografía}


AlBelda MARCO, Marta (2010). «¿Cómo se reconoce la atenuación?». (Des)cortesía en español, pp. 41-70.

BACH, K. (1998). Routledge Encyclopedia of Philosophy. Londres: Routledge.

BosQue, I. (1980). Las categorías gramaticales. Madrid: Arco Libros.

BRIZ, A. (1995). «La atenuación en la conversación coloquial. Una categoría pragmática». En Cortés, L. (ed.); El español actual. Almería: Ediciones Universidad de Almería.

CABedo, A. y S. Pons (2013). Corpus Valesco 2.0. Consultado de: www.valesco.es

CAFFI, Claudia (1999). «On mitigation». Journal of Pragmatics, no 31, 881-909.

CAFFI, Claudia (2007). Mitigation, Elsevier.

ChAnNell, J. (1985). «Vagueness as a conversational strategy». En Channell, J. (ed.); Nottingham Linguistic Circular, $\mathrm{n}^{\mathrm{o}}$ 14, 3-24.

Channell, J. (1994). Vague Language. Oxford: Oxford Universtity Press.

Cintula, P., C. Fermüller, L. Godo y P. HÁJeK (2011). Understanding vagueness: Logical, philosophical and linguistic perspectives. Londres: College Publications.

CRissmore, A. y W. VAN DE KOPPLE (1988). «Readers' learning from prose: The effects of hedges». Written Communication, 5, Vol. 2, 184-202.

EKLUND, M. (2001). «Supervaluationism, Vagueifiers, and Semantic Overdetermination». Dialectica, 55, 363-387.

EKLUND, M. (2005). «What Vagueness Consists in». International Journal for Philosophy in the Analytic Tradition, $\mathrm{n}^{\mathrm{o}}$ 1, Vol. 125, 27-60.

FARA, G. y G. RuSSELl (2012). The Routledge Companion to Philosophy of Language. Nueva York: Routledge.

Fraser, B. (1975). «Hedged performatives». En Cole, P. y Morgan, J. (eds.); Syntax and semantics, $\mathrm{n}^{\mathrm{o}} 3$ (187-210). New York: New York Academic Press.

FRASER, B. (1980). «Conversational mitigation». Journal of Pragmatics, 4, 341-50.

GRUPO VAL.Es.Co. (2002). «Corpus de conversaciones coloquiales». Oralia (anejos). Madrid: Arco Libros.

HidAlGO, A. (2013). «La fono(des)cortesía: marcas prosódicas (des)corteses en español hablado. Su estudio a través de corpus orales». RLA, 55, 127-50.

KALtenBÖCK, G. (2007). «Spoken parenthetical caluses in English. A taxonomy». En Dehé, N. y Kavalova, Y. (eds.): Parentheticals (pp.25-52). Amsterdam: John Benjamins Publishing. DOI: https://doi.org/10.1075/la.106

Kaltenböck, G., W. Mihatsch y S. Schneider (2010). New Approaches to Hedging. Amsterdam: Emerald Group Publishing.

KeEfe, R. (2003). Theories of Vagueness. Cambridge: Cambridge University Press.

KeEfe, R. y P. Smith (1997). Vagueness: A Reader. Cambridge: MIT Press.

LAKOFF, G. (1973). «Hedges: A study in meaning criteria and the logic of fuzzy concepts». Journal of Philosophical Logic 2, 458-508. 
LeVInson, S. (2000). Presumptive Meanings. The Theory of Generalized Conversational Implicature. Cambridge: MIT Press.

Minatsch, W. (2013). «Hedges». En Chappelle, C. (ed.); The Encyclopedia of Applied Linguistics (pp. 2457-2462). Oxford: Wiley-Blackwell.

SAdock, J. y Zwicky, A. (1975). «Ambiguity Tests and How to Fail Them». Syntax and Semantics. New York: New York Academic Press.

SEvi, A. (1998). A semantics for 'Almost' and 'Barely'. Tesis de máster. Tel-Aviv University.

SimON-VANDENBERGEN, A. M. (2000). «The functions of $I$ think in political discourse». International Journal of Applied Linguistics, 1, 41-63.

PONS, S. (2005). La enseñanza de la pragmática en el aula de E/LE. Madrid: Arco-Libros.

PRINCE, E. F., J. FRADER y C. BOSK (1982). «On hedging in physician-physician discourse». Linguistics and the Professions, 83-97.

VOGHERA, M. (2017). «La nascita delle costruzioni non nominali di specie, genere, sorta e tipo: uno studio basato su corpora». En D’Achille, P. y Grossmann, M. (eds.); Per la storia della formazione delle parole in italiano (pp. 277-307). Florencia: Franco Cesati Editore.

WACHTEL, T. (1980). «Pragmatic Approximations». Jounal of Pragmatics, 4, 201-211.

WiLLIAMSON, T. (1994) Vagueness. Londres: Routledge.

WiLliamson, T. (2007a). The Philosophy of Philosophy. Malden: Blackwell. 